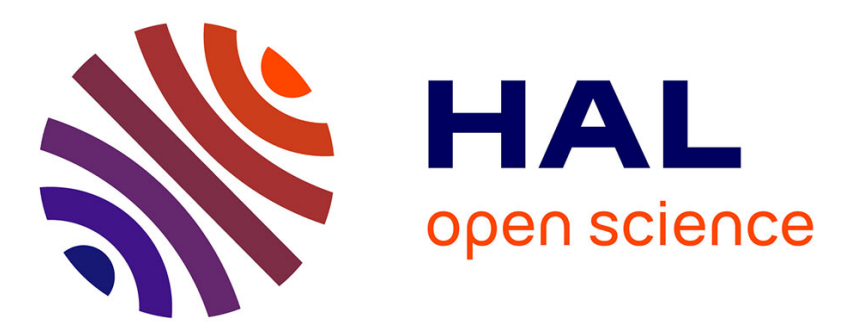

\title{
L'effet Raman et le pivotement des molécules dans les cristaux. Théorie générale et vérification expérimentale dans le cas du naphtalène
}

\author{
A. Kastler, A. Rousset
}

\section{- To cite this version:}

A. Kastler, A. Rousset. L'effet Raman et le pivotement des molécules dans les cristaux. Théorie générale et vérification expérimentale dans le cas du naphtalène. J. Phys. Radium, 1941, 2 (2), pp.49-57. 10.1051/jphysrad:019410020204900 . jpa-00233778

\section{HAL Id: jpa-00233778 https://hal.science/jpa-00233778}

Submitted on 1 Jan 1941

HAL is a multi-disciplinary open access archive for the deposit and dissemination of scientific research documents, whether they are published or not. The documents may come from teaching and research institutions in France or abroad, or from public or private research centers.
L'archive ouverte pluridisciplinaire HAL, est destinée au dépôt et à la diffusion de documents scientifiques de niveau recherche, publiés ou non, émanant des établissements d'enseignement et de recherche français ou étrangers, des laboratoires publics ou privés. 


\title{
LE JOURNAL DE PHYSIQUE
}

\section{LE RADIUM}

\section{L'EFFET RAMAN ET LE PIVOTEMENT DES MOLÉGULES DANS LES GRISTAUX. THÉORIE GÉNÉRALE ET VÉRIFIGATION EXPÉRIMENTALE DANS LE GAS DU NAPHTALĖNE}

\author{
Par A. KASTLER et A. ROUSSET. \\ Faculté des Sciences de Bordeaux (1).
}

\begin{abstract}
Sommaire. - On montre que les raies Raman de faible fréquence, découvertes par Gross et Vuks dans le spectre de la lumière diffusée par les cristaux organiques, sont dues à des pivotements cohérents des molécules du cristal. On calcule l'état de polarisation et l'intensité des vibrations diffusées par les molécules pivotantes d'un cristal. Les résultats de ce calcul sont confrontés avec les mesures de polarisation sur les spectres Raman du naphtalène et de la calcite. On rapporte, avec quelques détails, les résultats de l'étude expérimentale de la polarisation et de l'intensité des raies de faible fréquence d'un monocristal de naphtalène : ces résultats sont en excellent accord avec l'hypothèse de pivotements cohérents des molécules autour de leurs axes d'inertie. Enfin, les auteurs insistent sur les rapports nécessaires entre l'effet du pivotement des molécules dans les cristaux et l'effet de la rotation de ces mêmes molécules dans un liquide, révélés par la diffusion moléculaire de la lumière.
\end{abstract}

1. Théorie classique de la diffusion de la lumière par une molécule pivotante. - Soient $U$, $V, W$ les trois axes rectangulaires de l'ellipsoïde d'inertie d'une molécule; $g, g^{\prime}$ et $g^{\prime \prime}$ les réfractivités principales mesurées suivant ces mêmes axes (modèle moléculaire de Langevin). Nous supposons que la molécule exécute autour d'une orientation moyenne, définie par les axes fixes $U_{0}, V_{0}, W_{0}$, des oscillations pendulaires de faible amplitude (ou pivotements) de fréquence $u, v, w$.

Éclairons la molécule par la vibration lumineuse $\sin 2 \pi N t$ parallèle à $U_{0}$. Projetons la vibration incidente sur chacun des axes de la molécule pivotante. En multipliant l'amplitude de chaque projection par la réfractivité correspondante, nous obtenons les projections du moment induit suivant ces mêmes axes. Les amplitudes des vibrations diffusées, parallèles à chacun des axes fixes $U_{0}, V_{0}, W_{0}$, sont égales aux projections de ce moment induit sur ces axes.

I ${ }^{\circ}$ La molécule pivote autour de $U_{0}$. - Aucune modification de fréquence dans le moment induit qui reste couché sur $U_{\mathbf{0}}$.

(1) Exposé dans une conférence de A. Rousset devant la Société française de Physique (séances de Pentecôte 1941). $2^{\circ}$ La molécule pivote autour de $V_{0}$ (fig. I). Les positions des axes $U$ et $W$ sont à chaque instant fixées par l'angle $\alpha=\alpha_{0} \sin 2 \pi v t$. Suivant les axes de la molécule, les projections du moment induit s'écrivent

$$
\begin{aligned}
& \text { Sur } \mathrm{U} \ldots \ldots g \cos \alpha \sin 2 \pi N t \\
& " V \ldots \ldots o \\
& \text { " W..... - } g^{\prime \prime} \sin \alpha \sin 2 \pi N t
\end{aligned}
$$

d'où les projections de ce moment sur les axes fixes $U_{0}, V_{0}, W_{0}$ :

$$
\begin{array}{ccl}
\text { Sur } & \mathbf{U}_{0} \ldots \ldots & \left(g \cos ^{2} \alpha-g^{\prime \prime} \sin ^{2} \alpha\right) \sin 2 \pi N t \\
\Rightarrow & \mathbf{V}_{0} \ldots \ldots & o \\
\Rightarrow & \mathbf{W}_{0} \ldots \ldots & \left(g-g^{\prime \prime}\right) \sin \alpha \cos \alpha \sin 2 \pi N t
\end{array}
$$

Mais $\alpha_{0}$ et par conséquent $\alpha$ sont petits. On peut poser $\cos \alpha=1 ; \sin ^{2} \alpha=0$ et $\sin \alpha=\alpha=\alpha_{0} \sin 2 \pi v t$. Il vient alors

$$
\begin{array}{cccc}
\text { Projection } & \text { sur } & \mathbf{U}_{0} \ldots \ldots \ldots & g \sin 2 \pi N t \\
" & " & \mathbf{V}_{0} \ldots \ldots \ldots & 0 \\
" & " & \mathbf{W}_{0} \ldots \ldots \ldots & \alpha_{0}\left(g-g^{\prime \prime}\right) \sin 2 \pi i t \sin 2 \pi N t
\end{array}
$$

Le moment des fréquences modifiées est couché sur $W_{0}$; on peut l'écrire

$$
\frac{\mathrm{I}}{2} \alpha_{0}\left(\because-g^{\prime \prime}\right)[\cos 2 \pi(\lambda-v) t-\cos 2 \pi(\boldsymbol{V}+v) t]
$$


Son amplitude, pour chacune des deux fréquences $N \pm v$, vaut $\frac{1}{2} \alpha_{0}\left(g-g^{\prime \prime}\right)$.

$3^{\circ}$ La molécule pivote autour de $W_{0}$. - Dans ce cas, les doublets de Hertz de fréquences $\mathrm{N} \pm w$ sont couchés sur $V_{0}$ et leur amplitude vaut $\frac{\mathrm{I}}{2} \alpha_{0}\left(g-g^{\prime \prime}\right)$.

Ce calcul montre que les intensités des raies de pivotement varient comme le carré de l'anisotropie optique de la molécule, que

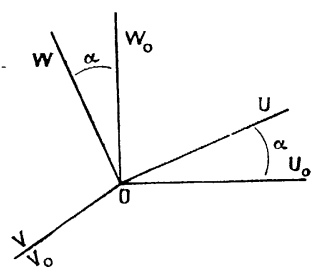

Fig. I . seules sont efficaces les vibrations excitatrices perpendiculaires à l'axe de pivotement et que le moment induit dans la molécule est perpendiculaire à l'axe de pivotement.

Dès i 935 , l'un de nous [I] avait tiré de ce calcul l'intensité et la polarisation des radiations diffusées par des molécules pivotantes orientées au hasard (liquides à molécules polaires par exemple); l'intensité lumineuse diffusée, proportionnelle au carré de l'amplitude maximum, n'est qu'une faible fraction du spectre de rotation de la molécule libre de tourner.

Raman et Negundaki [2], en précisant la direction du moment induit dans le cas d'ions de même orientation moyenne, ont donné une interprétation très simple des variations d'intensité que présentent certaines raies Raman d'un cristal de nitrate de sodium quand on change l'orientation du cristal par rapport à la vibration incidente et à la direction d'observation. Ils admettent que dans le spectre Raman du nitrate de sodium cristallisé, les raies de faible fréquence $\left(^{2}\right)$ I $85 \mathrm{~cm}^{-1}$ et $9^{8} \mathrm{~cm}^{-1}$ sont dues à des oscillations de l'ion $\mathrm{NO}_{3}^{-}$autour d'un axe du plan équatorial. Ils vérifient alors, conformément aux résultats du calcul rappelés plus haut, que ces raies disparaissent dans les spectres où les fréquences internes $1065 \mathrm{~cm}^{-1}$ et $1375 \mathrm{~cm}^{-1}$ paraissent avec le maximum d'intensité ${ }^{3}$ ). En supposant ces pivotements quantifiés, les auteurs hindous ont calculé l'amplitude maximum en fonction du moment d'inertie $I$ et de la fréquence $v$ de pivotement. On obtient immédiatement, pour l'amplitude du moment diffusé, $\frac{1}{2}\left(g-g^{\prime \prime}\right) \sqrt{\frac{h}{2 \pi I v}}$.

Afin d'étendre à d'autres cristaux cette interprétation des raies Raman de faible fréquence, nous

(2) La présence dans le spectre Raman du nitrate de sodium, comme dans celui du spath calcaire, de deux raies de faible fréquence ayant les mêmes caractères de polarisation, reste à expliquer.

( ${ }^{3}$ ) On sait cependant (Cabannes et Canals, Comptes rendus, I $93 \mathrm{I}, 193$, p. 289) que les fréquences internes $\mathrm{r} 065 \mathrm{~cm}^{-1}$ et $1385 \mathrm{~cm}^{-1}$, comme les fréquences correspondantes $1087 \mathrm{~cm}^{-1}$ et $1437 \mathrm{~cm}^{-1}$ du spath, ne présentent pas les mêmes caractères de polarisation. allons traiter le cas plus général de la diffusion par les molécules pivotantes dont les axes d'inertie sont placés de façon absolument quelconque par rapport aux éléments de symétrie du cristal, éléments qui imposent la direction des vibrations excitatrices et la direction d'observation.

Soient $U, V, W\left(^{4}\right)$ les trois axes rectangulaires de l'ellipsoïde d'inertie autour duquel la molécule (ou l'ion) pivote avec la fréquence $u, v$ ou $w ; g, g^{\prime}$ et $g^{\prime \prime}$ les réfractivités principales de la molécule (ou de l’ion) relatives à ces mêmes axes. Soit un trièdre, lié aux éléments de symétrie du cristal, d'axes I, 2, 3.

Tableau des cosinus directeurs.

\begin{tabular}{|c|c|c|c|}
\hline & 1. & 2. & 3. \\
\hline $\mathrm{I} \ldots \ldots \ldots \ldots$ & $a_{1}$ & $a_{2}$ & $a_{3}$ \\
$\mathrm{~V} \ldots \ldots \ldots \ldots$ & $b_{1}$ & $b_{2}$ & $b_{3}$ \\
$\mathrm{~W} \ldots \ldots \ldots \ldots$ & $c_{1}$ & $c_{2}$ & $c_{3}$ \\
\hline
\end{tabular}

I ${ }^{\circ}$ Le vecteur électrique $\vec{E}$ de l'onde incidente est parallèle à l'axe 1 . - Les projections $E_{\mathrm{U}}, E_{\mathrm{V}}, E_{\mathrm{w}}$ sur les axes $U, V, W$ de la molécule sont respectivement proportionnelles à $a_{1}, b_{1}$ et $c_{1}$. Pour chacune de ces projections et pour chacun des pivotements autour de $U, V$ et $W$, appliquons les résultats du calcul précédent. Les amplitudes des moments induits $\vec{M}_{\mathrm{E}}$, $\vec{M}_{\mathrm{v}}$ et $\vec{M}_{\mathrm{w}}$ de fréquences $N_{-\mathrm{v}}^{+}, N_{-\mathrm{v}}^{+}, N_{-\mathrm{w}}^{+}$et leur direction sont données, dans chaque cas, par le tableau ci-dessous (*) :

\begin{tabular}{|c|c|c|c|}
\hline & $\overrightarrow{M_{\mathrm{c}}}$ & $\overrightarrow{M_{\mathrm{r}}}$. & $\overrightarrow{M \mathrm{w}}$ \\
\hline$E_{\mathrm{V}} \ldots \ldots$ & $o$ & $\begin{array}{c}/ / \text { à W } \\
a_{1}\left(g^{\prime \prime}-g\right)\end{array}$ & $\begin{array}{c}/ / \grave{\mathrm{a}} \mathbf{V} \\
a_{1}\left(g-g^{\prime}\right)\end{array}$ \\
\hline$E_{\mathrm{V}} \ldots \ldots$ & $\begin{array}{c}M / \grave{a ̀ ~ W} \\
b_{1}\left(g^{\prime}-g^{\prime \prime}\right)\end{array}$ & 0 & $\begin{array}{c}/ / \text { à U } \\
b_{1}\left(g-g^{\prime}\right)\end{array}$ \\
\hline$E_{\mathbb{W}} \ldots$ & $\begin{array}{c}/ / \grave{a ̀ ~ V} \\
c_{1}\left(g^{\prime}-g^{\prime \prime}\right)\end{array}$ & $\begin{array}{c}/ / \text { à } \mathrm{L} \\
c_{1}\left(g^{\prime \prime}-g\right)\end{array}$ & $o$ \\
\hline
\end{tabular}

Si le vecteur électrique $\vec{E}$ est parallèle à l'axe 2 ou à l'axe 3 , il suffit de remplacer, dans le tableau ci-dessus, l'indice $\mathrm{x}$ par l'indice 2 ou 3 . On obtient alors, pour chacune des fréquences Raman de pivotement ( $u$, par exemple), le tenseur $(U)$ qui

(4) On peut, sans ambiguïté, désigner ici et dans la suite des calculs, les directions moyennes des axes de la molécule pivotante par $U V W$ et non plus par $U_{0} V_{0} W_{0}$.

(*) Le symbole " / à $U$ " signifie " parallèle à $U$ ". 
relie le vecteur électrique $\vec{E}$ de l'onde incidente et le moment induit $\vec{M}_{\mathrm{v}}$ qui émet la raie Raman, rapportés aux axes $1,2,3$ [tous les termes doivent être multipliés par $\left.\left(g^{\prime}-g^{\prime \prime}\right)\right]$.

\begin{tabular}{|c|c|c|c|}
\hline$(\mathrm{U})$. & $E_{1}$. & $E_{2}$. & $E_{3}$. \\
\hline$M_{1} \ldots$ & $\begin{array}{c}2 b_{1} c_{1} \\
\boldsymbol{M}_{1} \ldots \ldots\end{array}$ & $\begin{array}{c}\left(b_{1} c_{2}+b_{2} c_{1}\right) \\
2 b_{2} c_{2}\end{array}$ & $\begin{array}{c}\left(b_{1} c_{3}+b_{3} c_{1}\right) \\
\left(b_{2} c_{3}+b_{3} c_{2}\right) \\
2 b_{3} c_{3}\end{array}$ \\
\hline$M_{3} \ldots \ldots$ & $b_{1} c_{3}+b_{3} c_{1}$ & $\left(b_{2} c_{3}+b_{3} c_{2}\right)$ & 2 \\
\hline
\end{tabular}

Les tenseurs $(V)$ et $(W)$, relatifs à $\vec{M}_{\mathrm{v}}$ et $\vec{M}_{\mathrm{w}}$, se déduisent du tenseur précédent par une permutation circulaire de $a, b, c$, et de $g, g^{\prime}, g^{\prime \prime}$.

2. Calcul des tenseurs dans le cas de pivotements cohérents des molécules d'un cristal monoclinique. - Prenons la maille d'un cristal monoclinique comme le naphtalène qui renferme deux molécules (I et II) orientées symétriquement par rapport au plan de symétrie (oro) normal à l'axe binaire (axe 2).

Supposons cohérents tous les pivotements des molécules du cristal. Le pivotement d'ensemble des molécules I et II autour de leur axe U constitue, pour la maille, une vibration symétrique $U_{\mathbf{s}}$ ou antisymétrique $U_{\mathrm{A}}$ par rapport à son plan de symétrie, suivant que les pivotements de ces deux molécules sont symétriques ou antisymétriques par rapport à ce plan $\left(^{5}\right)$. Au point de vue de son orientation, nous savons que la molécule II peut être considérée comme l'image, par réflexion, de la molécule I, dans le plan de symétrie. Nous obtenons son tenseur $\left(U_{\mathrm{II}}\right)$ en changeant, dans le tenseur ci-dessus, les signes de $a_{2}, b_{2}$ et $c_{2}$ correspondant aux projections sur l'axe binaire, d'où la forme des tenseurs d'amplitudes pour les deux molécules :

\begin{tabular}{|c|c|c|c|c|c|c|c|}
\hline \multicolumn{4}{|c|}{ Molécule 1.} & \multicolumn{4}{|c|}{ Molécule II. } \\
\hline$\left(U_{1}\right)$ & $E_{1}$. & $E_{2}$ & $E_{3}$ & $\left(U_{\mathrm{HI}}\right)$. & $E_{1}$. & $E_{2}$. & $E_{3}$. \\
\hline$M_{1}$. & $A_{11}$ & $A_{12}$ & $A_{13}$ & $M_{1}$. & $A_{11}$ & $-A_{12}$ & $A_{13}$ \\
\hline$M_{2} \ldots$ & $A_{21}$ & $A_{22}$ & $A_{23}$ & $M_{2}$. & $-A_{21}$ & $A_{22}$ & $-A_{23}$ \\
\hline$M_{3} \ldots$ & $A_{31}$ & $A_{3 \cong}$ & $A_{33}$ & $M_{3} .$. & $A_{31}$ & $-A_{32}$ & $A_{33}$ \\
\hline
\end{tabular}

Le couplage des pivotements des deux molécules en vibration symétrique et antisymétrique revient à un ajustement différent des phases des pivotements des deux molécules I et II. La combinaison symé-

( $\left.{ }^{5}\right)$ Ces pivotements sont en même temps symétriques ou antisymétriques par rapport à l'axe binaire. trique est due à deux pivotements en concordance de phase; les deux tenseurs d'amplitudes s'ajoutent :

$$
\left(U_{S}\right)=\left(U_{\mathrm{I}}\right)+\left(U_{\mathrm{II}}\right)
$$

La combinaison antisymétrique est due à deux pivotements en opposition de phase; dans ce cas, une augmentation de $\pi$ pour la phase change le signe de tous les moments induits du tenseur $\left(U_{\mathrm{II}}\right)$; les deux tenseurs d'amplitudes se retranchent :

$$
\left(U_{A}\right)=\left(U_{\mathrm{I}}\right)-\left(U_{\mathrm{II}}\right) \text {. }
$$

On obtient ainsi, pour les vibrations $U_{\mathbf{S}}$ et $U_{\mathrm{A}}$, les deux tenseurs complémentaires ci-dessous $\left(^{6}\right)$ :

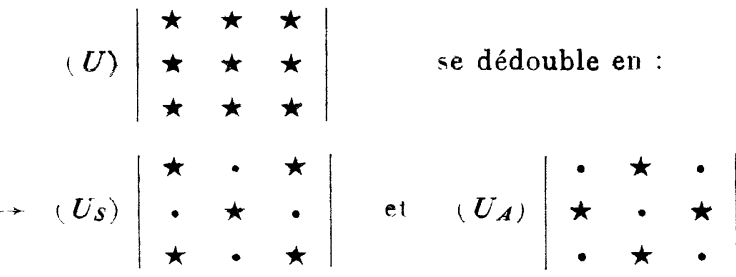

Nous retrouvons pour nos pivotements les résultats généraux établis par J. Cabannes [3] pour les oscillations symétriques et antisymétriques des cristaux du système monoclinique.

Les deux tenseurs d'intensités, dont les termes s'obtiennent en élevant au carré les termes correspondants des tenseurs d'amplitude, sont donc également complémentaires et n'ont aucun terme commun. Nous les reporterons dans la suite dans un même cadre.

Les deux modes de couplage, symétrique et antisymétrique, quoique relatifs à un même pivotement, mettent en jeu des forces de rappel légèrement différentes : par suite, aux vibrations symétriques et antisymétriques de la maille doivent correspondre des fréquences de pivotement légèrement différentes. Dans le cas général de la molécule de Langevin à trois réfractivités inégales, il faut donc prévoir six raies " externes " différentes, voisines de la raie excitatrice : $U_{\mathrm{S}}$ et $U_{\mathrm{A}}, V_{\mathrm{S}}$ et $V_{\mathrm{A}}, W_{\mathrm{S}}$ et $W_{\mathrm{A}}$, groupées en trois doublets. A chacune des composantes d'un doublet, $U_{\mathrm{S}}$ ou $U_{\mathrm{A}}$ par exemple, correspond l'un des tenseurs complémentaires $\left(U_{\mathrm{S}}\right)$ ou $\left(U_{\mathrm{A}}\right)$ formé de termes soit uniquement symétriques, soit uniquement antisymétriques.

3. Application au naphtalène. - La molécule du naphtalène est plane. Désignons par $U$ son grand axe (ou ligne joignant les centres des noyaux benzéniques), par $V$ son petit axe (perpendiculaire à $\mathrm{U}$ et situé dans le plan moléculaire), par W la normale au plan moléculaire (fig. 2). On connaît, par l'étude aux rayons X (Robertson) ['́l, l'orien-

${ }^{(6)}$ Le tenseur ( $\left.U_{s}\right)$ ne contient que les termes $A_{\alpha s}$ dont la somme des indices $\alpha+\beta$ est paire le tenseur; $\left(U_{A}\right)$ ne contient que les termes dont la somme des indices est impaire. 
tation des axes $\mathrm{U}, \mathrm{V}, \mathrm{W}$ par rapport au trièdre constitué par l'arête $a$ et $b$ de la maille clinorhombique ( $b$, axe binaire ou axe 2 ) et la perpendiculaire $c^{\prime}$ au plan des arêtes $a$ et $b$ (plan oor) de la maille.

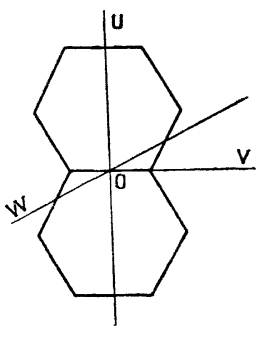

Fig. 2.

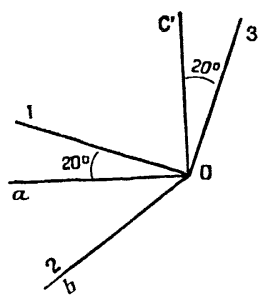

Fig. 3.
Voici le tableau des cosinus directeurs :

TableaU I.

\begin{tabular}{|c|c|c|c|}
\hline & $a$. & $\begin{array}{c}b \\
\text { (axe 2). }\end{array}$ & $c^{\prime}$. \\
\hline $\mathbf{U} \ldots$ & $-0,43$ & $-0,22$ & 0,88 \\
\hline V... $\ldots$ & $o, 32$ & 0,88 & 0,36 \\
\hline$W \ldots \ldots \ldots$ & $-0,84$ & 0,43 & $-0,32$ \\
\hline
\end{tabular}

On en déduit facilement les cosinus directeurs des mêmes axes $\mathrm{U}, \mathrm{V}, \mathrm{W}$ par rapport au trièdre $\mathrm{I}, 2,3$ des axes de l'ellipsoïde des indices : les axes 1 et 3 sont situés dans le plan de symétrie $a c^{\prime}$, l'axe 3 est confondu avec l'arête du dièdre des plans moléculaires [5], donc incliné de $20^{\circ} \operatorname{sur} c^{\prime}$ (fig. 3).

TABLEAU II.

\begin{tabular}{|c|c|c|c|}
\hline & 1. & 2. & 3. \\
\hline $\mathrm{L}^{-} \ldots \ldots$ & $-0, I_{1}$ & $-0,22$ & 0,97 \\
\hline V....... & $o, 42$ & 0,88 & 0,23 \\
\hline W....... & $-o, 9^{\circ}$ & 0.43 & o \\
\hline
\end{tabular}

Le monocristal de naphtalène utilisé dans nos recherches a la forme d'un parallélépipède rectangle dont les arêtes sont parallèles aux axes $a, b, c^{\prime}$ de Robertson ( ${ }^{7}$ ). Soit OXYZ un trièdre fixe dans l'espace, $\mathrm{OZ}$ étant vertical. Le cristal est placé en $\mathrm{O}$ avec ses arêtes respectivement parallèles à XYZ. On éclaire le long de OX par un faisceau de lumière polarisée suivant $\mathrm{OY}$ ou suivant $\mathrm{OZ}$. Les vibrations diffusées dans la direction OY sont analysées par un biré-

(7) Ill aurait été préfèrable d'étudier un parallélépipède taillé suivant les arêtes 1, 2, 3 de l'ellipsoïde des indices, mais nous ne disposions pas d'un bel échantillon de ce genre. fringent (wollaston) de section principale verticale, de façon à donner sur la fente du spectrographe deux images séparées du cristal, l'une $i$ contenant les vibrations diffusées parallèles à $O X$, l'autre $I$ les vibrations diffusées parallèles à $\mathrm{OZ}\left(^{8}\right)$.

Comme les arêtes de notre cristal ne coïncident pas exactement avec les axes des indices principaux (sauf pour l'axe binaire), la biréfringence va introduire une complication : c'est seulement dans le cas où le plan de symétrie est horizontal que les vibrations incidentes ou diffusées restent parallèles aux arêtes du cristal. Si l'on éclaire (ou si l'on observe) à travers le plan de symétrie, les vibrations incidentes (ou diffusées) qui se propagent à travers le cristal sont parallèles aux axes 1 et 3 , il en résulte qu'en général les intensités et les polarisations expérimentales doivent présenter un léger écart avec les valeurs calculées à partir des cosinus directeurs, tant du Tableau I relatif au trièdre $a b c^{\prime}$ que du Tableau II relatif au trièdre $1,2,3$.

Dans les tenseurs d'intensité ci-dessous, nous avons reporté les intensités calculées en confondant le trièdre XYZ successivement avec les deux trièdres $a b c^{\prime}$ et I, 2, 3. Dans chaque case le nombre du haut se rapporte au trièdre $a b c^{\prime}$, celui du bas au trièdre $\mathrm{I}, 2,3\left({ }^{9}\right)$.

Les intensités données par l'expérience doivent être comprises entre ces valeurs limites. Les intensités expérimentales évaluées qualitativement sur les clichés et exprimées par les lettres $F$ (forte), $m$ (moyenne), $f$ (faible), o (nulle) ont été reportées dans le même cadre ${ }^{\left({ }^{10}\right)}$.

Les termes expérimentaux ont été obtenus de la manière suivante : on a donné successivement au cristal les six orientations différentes possibles par rapport au trièdre fixe $\mathrm{XYZ}$ :

$\begin{array}{lllllll}\mathbf{X} \ldots \ldots & 3 & \text { I } & \text { I } & 3 & 2 & \mathbf{2} \\ \mathrm{Y} \ldots \ldots & \mathrm{I} & 3 & 2 & \mathbf{2} & \mathrm{I} & 3 \\ \mathrm{Z} \ldots \ldots & 2 & 2 & 3 & \text { I } & 3 & \text { I }\end{array}$

Pour chacune de ces six orientations, le vecteur électrique de la lumière excitatrice a été dirigé d'abord parallèlement à l'axe $\mathrm{OZ}\left(E_{z}\right)$, ensuite parallèlement à l'axe OY $\left(E_{y}\right)$. Les 12 cas correspondants sont numérotés de 1 à 12 dans la première colonne de la planche I. Enfin, le biréfringent placé

${ }^{(8)}$ Une bilame demi-onde placée devant la fente rend les deux vibrations issues du wollaston toutes deux normales aux arêtes des prismes du spectrographe. Dans ces conditions, les coefficients de transmission sont égaux et optima pour ces deux vibrations.

$\left({ }^{9}\right)$ Chaque terme est à multiplier : pour le tenseur $(U)$, par $\left(g^{\prime}-g^{\prime \prime}\right)^{2}$, pour le tenseur $(V)$, par $\left(g-g^{\prime \prime}\right)^{2}$ et pour le tenseur $(\mathrm{IV})$, par $\left(g-g^{\prime}\right)^{2}$.

${ }^{\left({ }^{10}\right)}$ Les deux tableaux complémentaires relatifs aux pivotements autour d'un même axe $U$ par exemple, sont reportés dans le même cadre; pour chacune des vibrations $U_{s}$ et $U_{\mathrm{A}}$, on ne prend évidemment que les termes non nuls des tenseurs correspondants $\left(U_{S}\right)$ et $\left(U_{A}\right)$. 
D

sur le faisceau diffusé fournit, pour chacun de ces cas, deux spectres : spectre $i$, dû à la vibration diffusée parallèle à $\mathrm{OX}$, spectre $I$ dû à la vibration diffusée parallèle à $\mathrm{OZ}$. Chacun de ces 24 spectres correspond à un terme déterminé $\left(A_{\alpha \beta}\right)^{2}$ des tenseurs d'intensité. Deux colonnes de la planche I indiquent : l'une la notation du terme correspondant à chaque spectre, l'autre son caractère de symétrie ( $s$, symétrique; $a$, antisymétrique). Dans les deux colonnes suivantes on donne une représentation schématique des spectres permettant de confronter les prévisions théoriques et les résultats expérimentaux : l'épaisseur des traits matérialise l'intensité des raies correspondantes. Les points d'interrogation correspondent à des raies non décelables à cause d'un fond continu qui les recouvre. Certains termes faibles $\left[\left(A_{13}\right)^{2}\right.$ de $(W)$ et de $(U)]$ ne paraissent pas toujours sur les clichés, le temps de pose ayant été sans doute insuffisant. Mais, dans l'ensemble, l'accord est frappant lorsqu'on adopte l'attribution suivante pour les six raies voisines de la raie excitatrice du spectre de diffusion du naphtalène-cristal [6] :

$$
\begin{array}{cccc}
46 \mathrm{~cm}^{-1}, & 54 \mathrm{~cm}^{-1}, & 74 \mathrm{~cm}^{-1}, & 76 \mathrm{~cm}^{-1} \\
W_{A} & W_{S} & V_{A} & V_{S} \\
& 109 \mathrm{~cm}^{-1}, & 127 \mathrm{~cm}^{-1} . \\
U_{S} & U_{A}
\end{array}
$$

Le doublet 46-54 $\mathrm{cm}^{-1}$ correspond donc aux pivotements cohérents des molécules autour de la normale $\mathrm{W}$ au plan moléculaire, le doublet étroit $74-76 \mathrm{~cm}^{-1}$ aux pivotements autour du petit axe $\mathrm{V}$, et enfin le doublet $109-127 \mathrm{~cm}^{-1}$ aux pivotements autour $d u$ grand

\begin{tabular}{|c|c|c|c|c|c|c|c|c|c|}
\hline & \multicolumn{3}{|c|}{$(U)$} & \multicolumn{3}{|c|}{$(V)$} & \multicolumn{3}{|c|}{$(W)$} \\
\hline & $E_{1}$ & $E_{2}$ & $E_{3}$ & $E_{1}$. & $E_{2}$ & $E$. & $E_{1}$ & $E_{2}$ & $E_{3}$. \\
\hline$\left(M_{1}\right)^{2} \ldots \ldots$ & 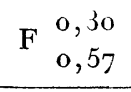 & $\begin{array}{r}0,36 \\
\quad 0,37 \\
\end{array}$ & $\begin{array}{r}0,16 \\
0,04 \\
\end{array}$ & f $\begin{array}{l}0,52 \\
0,03\end{array}$ & $f \begin{array}{l}0 \\
0,02 \\
\end{array}$ & $\mathbf{F} \begin{array}{r}0,36 \\
0,76 \\
\end{array}$ & $0 \begin{array}{l}0,07 \\
0,01\end{array}$ & $\mathrm{~m} \begin{array}{l}o, 20 \\
0,03\end{array}$ & $\begin{array}{l}\mathrm{f} \quad, 02 \\
0,15\end{array}$ \\
\hline$\left(M_{2}\right)^{2} \ldots$ & $\mathbf{m} \begin{array}{l}\mathbf{0}, 36 \\
\mathbf{o}, 37 \\
\end{array}$ & $\mathrm{~F} \begin{array}{l}0,57 \\
0,57\end{array}$ & $\begin{array}{l}0,02 \\
0,01\end{array}$ & $f^{f} \begin{array}{l}o \\
0,02\end{array}$ & f $\begin{array}{l}0,04 \\
0.04\end{array}$ & $\begin{array}{r}0,20 \\
0,17 \\
\end{array}$ & $\begin{array}{r}0,20 \\
0,03 \\
\end{array}$ & $\mathrm{~m}_{0,15}^{0.15}$ & 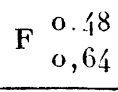 \\
\hline$\left(M_{3}\right)^{2}:$ & f $\begin{array}{l}0,16 \\
0,04\end{array}$ & $\begin{array}{l}0,02 \\
0,01\end{array}$ & $\begin{array}{l}0,05 \\
0 \\
0\end{array}$ & $\mathrm{~F} \begin{array}{l}0,036 \\
0,76\end{array}$ & $\mathrm{~m} \begin{array}{l}0,20 \\
0, \mathrm{I} z\end{array}$ & $\mathbf{m}_{0}^{0,32}$ & f $\begin{array}{l}0,02 \\
o, 15\end{array}$ & 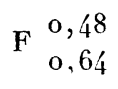 & $\mathrm{m} \begin{array}{l}0,39 \\
0,20\end{array}$ \\
\hline
\end{tabular}
axe $\mathrm{U}$ des molécules.

Tensetro d'Intensité.

L'ordre des fréquences est conforme à celui des moments d'inertie (calculés en négligeant la masse des atomes d'hydrogène) :

$$
\begin{aligned}
& I_{U}=2,2 \cdot \mathrm{IO}^{-38} \mathrm{~g} \cdot \mathrm{cm}^{2}, \\
& I_{V}=6,0 \cdot \mathrm{IO}^{-38} \mathrm{~g} \cdot \mathrm{cm}^{2}, \\
& I_{W}=8,4 \cdot \mathrm{IO}^{-38} \mathrm{~g} \cdot \mathrm{cm}^{2} .
\end{aligned}
$$

On vérifie facilement que les raies symétriques (54, 76 et $\left.109 \mathrm{~cm}^{-1}\right)$ et les raies antisymétriques (45, 74 et $127 \mathrm{~cm}^{-1}$ ) n'apparaissent jamais les unes et les autres dans un même spectre. On peut remarquer que les 24 spectres obtenus se ramènent à six types différents qui correspondent aux six termes différents des tenseurs d'intensité :

$$
\begin{aligned}
& \left(A_{11}\right)^{2}=I_{7}=I_{11}, \\
& \left(A_{22}\right)^{2}=I_{1}=I_{33}, \\
& \left(A_{33}\right)^{2}=I_{0}=I_{9 .} . \\
& \left(A_{12}\right)^{2}=I_{2}=i_{3}=i_{6}=I_{8}=i_{10}=i_{11}, \\
& \left(A_{23}\right)^{2}=i_{1}=I_{4}=I_{6}=i_{8}=i_{9}=i_{12} . \\
& \left(A_{13}\right)^{2}=i_{2}=i_{4}=i_{5}=i_{7}=I_{10}=I_{12} .
\end{aligned}
$$

L'examen des clichés montre la similitude des spectres d'un même groupe. Nous avons reproduit (pl. II) les meilleurs spectres de chaque type obtenus avec une dispersion plus grande. Nous ajoutons également les spectres obtenus en lumière incidente naturelle lorsque l'axe binaire du cristal est parallèle au faisceau incident $\mathrm{OX}$ : $\operatorname{cas}(9+\mathrm{I} 0)$ et $(\mathrm{I}$ I + I 2$)$. Le passage d'un de ces cas à l'autre correspond à la rotation du cristal autour de l'axe binaire. Pendant cette opération, le spectre $i$ contenant les raies antisymétriques reste inchangé $\left[i=\left(A_{12}\right)^{2}+\left(A_{23}\right)^{2}\right]$, tandis que le spectre $I$ contenant les raies symétriques se modifie [3],

$$
\boldsymbol{I}_{(9+10\}}=\left(A_{13}\right)^{2}+\left(A_{: 33}\right)^{2} \quad \text { et } \quad \boldsymbol{I}_{(11+12)}=\left(A_{11}\right)^{2}+\left(A_{13}\right)^{2} .
$$

L'intensité relative des raies (le doublet $74-76 \mathrm{~cm}^{-1}$ est le plus intense) montre que la réfractivité moyenne de la molécule est dirigée suivant son petit axe $\mathrm{V}$. L'étude quantitative des intensités relatives des différentes raies permettra la comparaison des différences de réfractivité $g-g^{\prime}, g^{\prime}-g^{\prime \prime}$ et $g-g^{\prime \prime}$ de la molécule de naphtalène. C'est ainsi, par exemple, que dans le spectre $i$ du cliché $\left(\right.$ I $\left._{1}+\mathrm{I}_{2}\right)$ dû au terme $\left(A_{12}\right)^{2}+\left(A_{23}\right)^{2}$ les intensités des trois raies correspondent à o, $67\left(g-g^{\prime}\right)^{2}$ pour $W_{\mathrm{A}}=45 \mathrm{~cm}^{-1}$, $0,20\left(g-g^{\prime \prime}\right)^{2}$ pour $V_{\mathrm{A}}=74 \mathrm{~cm}^{-1}$ et $0,38\left(g^{\prime}-g^{\prime \prime}\right)^{2}$ pour $U_{\mathrm{A}}=127 \mathrm{~cm}^{-1}$. Le simple examen qualitatif 
PLANCHE I - Confrontation des prévisions théoriques et des résultats expérimentaux.

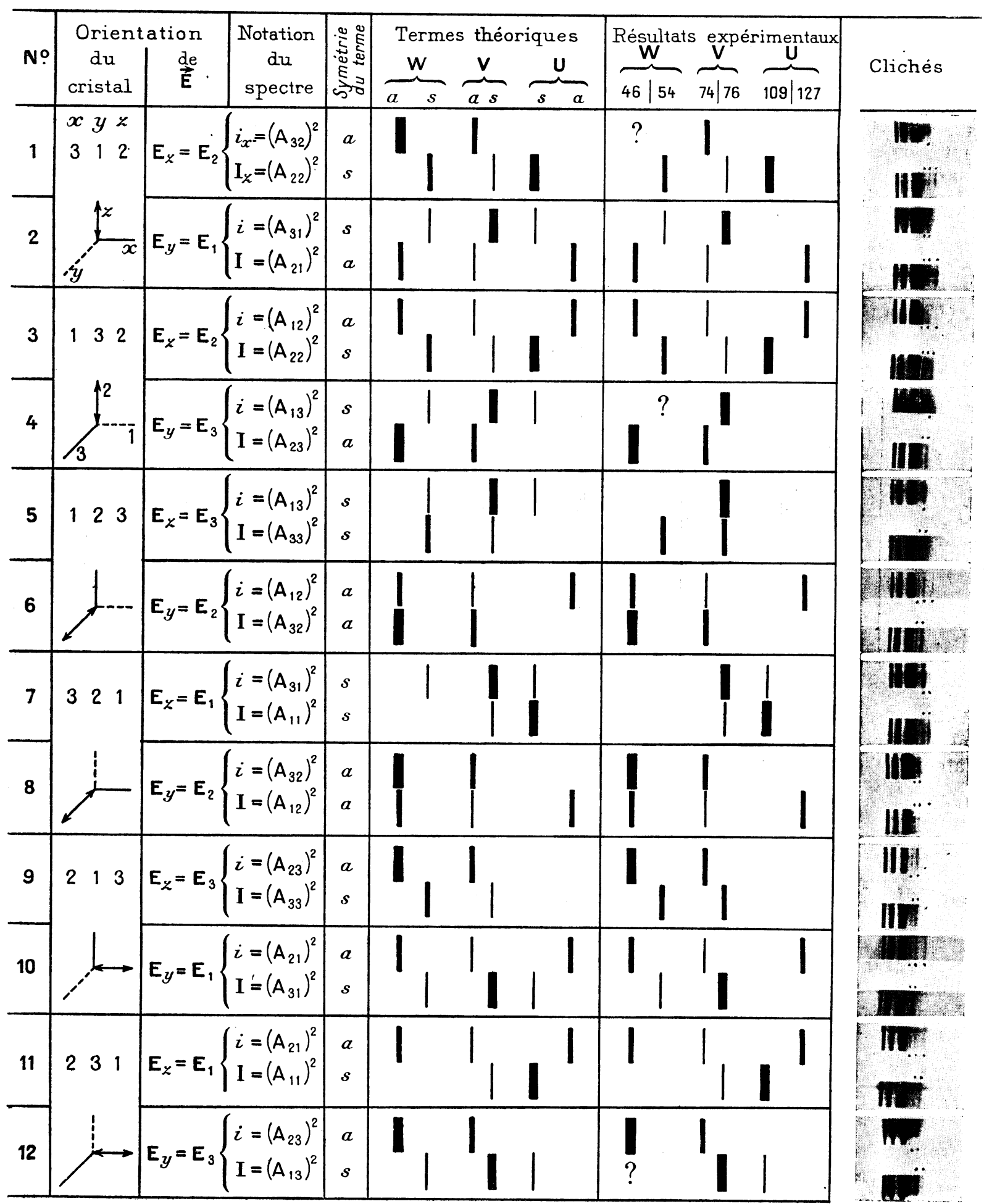




\section{PLANCHE II}

Reproduction de quelques spectres avec grande dispersion

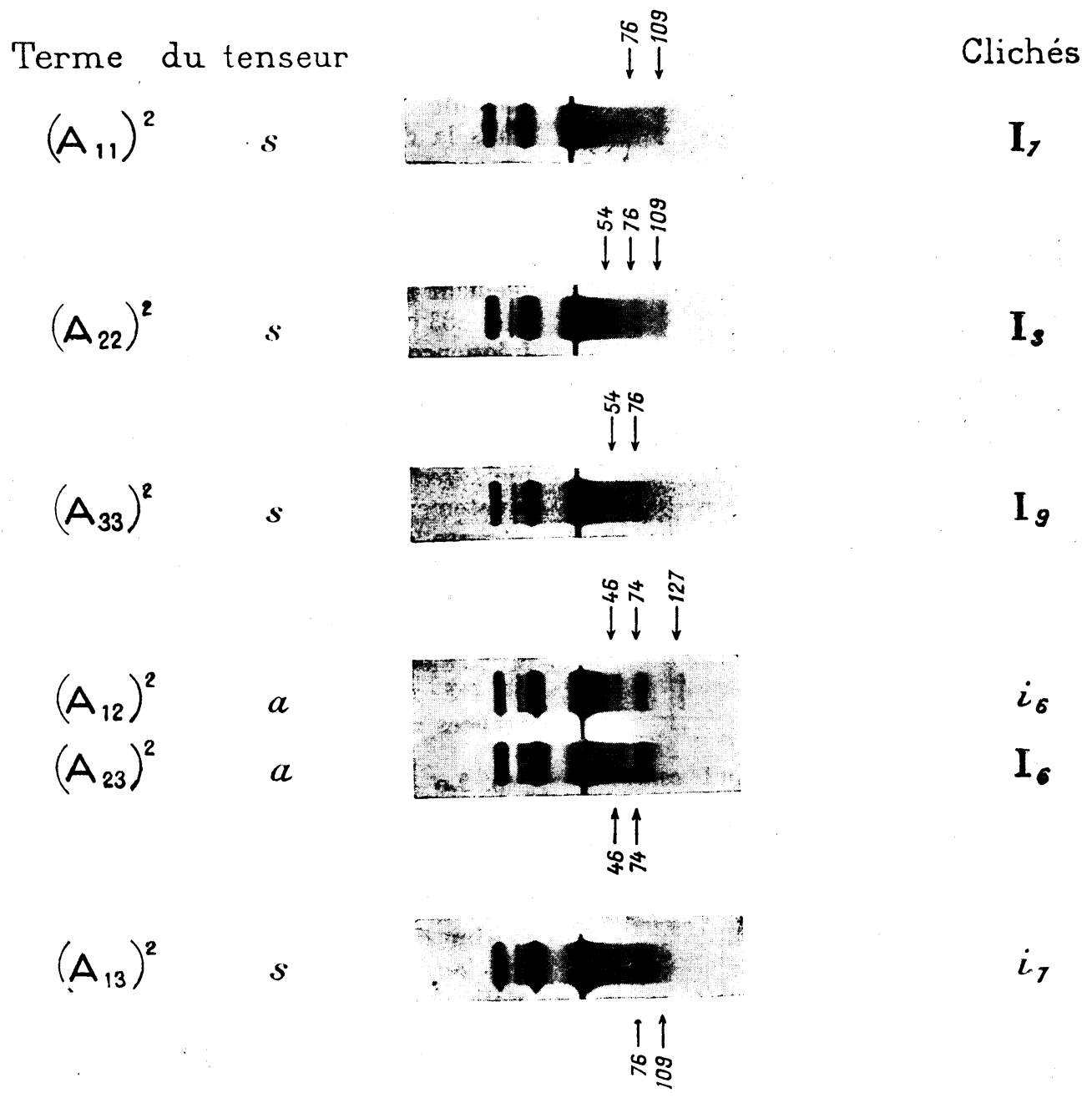

Excitation en lumière naturelle

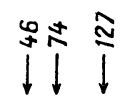

$\left(A_{12}\right)^{2}+\left(A_{23}\right)^{2}$

$\left(A_{13}\right)^{2}+\left(A_{23}\right)^{2}$

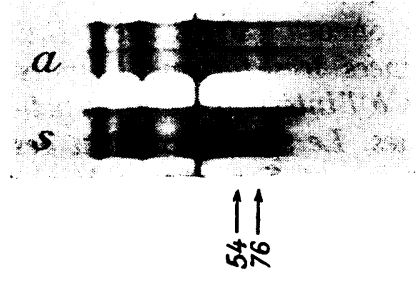

$(9+10)$

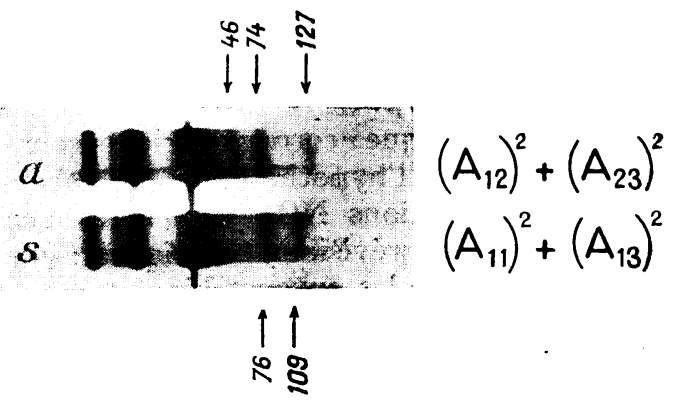

$(11+12)$ 
des clichés montre déjà que les différences $\left(g-g^{\prime}\right)$ et $\left(g^{\prime}-g^{\prime \prime}\right)$ sont du mème ordre de grandeur. L'étude photométrique nous a donné les résultats suivants :

$$
\left(g-g^{\prime}\right):\left(g^{\prime}-g^{\prime \prime}\right):\left(g-g^{\prime \prime}\right)=\mathrm{I}: \mathrm{I}, 3: 2,3 .
$$

4. Application à la calcite. - Nous avons rappelé que Raman et Negundaki ont vérifié leur théorie des pivotements sur les raies " externes" d'un cristal de nitrate de sodium. La maille renferme deux ions plans $\mathrm{NO}_{3}^{-}$, normaux à l'axe ternaire. Prenons cet axe du cristal à la fois pour l'axe W de l'ion $\mathrm{NO}_{3}^{-}$et pour l'axe 2 du trièdre attaché au cristal, chaque ion et le cristal dans son ensemble possédant au point de vue des réfractivités la symétrie de révolution autour de cet axe $\left(g=g^{\prime}\right)$. Si les pivotements des deux molécules se font autour de plusieurs axes situés dans leur plan équatorial et sont incohérents, on obtient le tenseur $(U+V)$ ci-dessous où tous les termes non nuls sont égaux :

\begin{tabular}{|c|c|c|c|}
\hline$(U+V) \ldots$ & $E_{1}$. & $E_{2 .}$ & $E_{3}$. \\
\hline$M_{1} \ldots \ldots$ & & $\star$ & $\cdot$ \\
$M_{2} \ldots \ldots$ & $\star$ & $\bullet$ & $\star$ \\
$M_{3} \ldots \ldots$ & & $\star$ & $\cdot$ \\
\hline
\end{tabular}

$\mathrm{Si}$, au contraire, ces pivotements sont cohérents et se couplent autour d'axes parallèles, à la vibration "symétrique " correspond un tenseur nul, à la vibra-
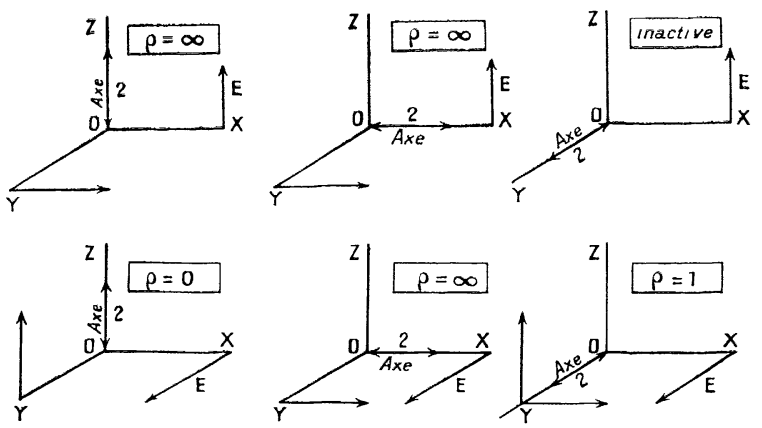

Fig. 4.

tion « antisymétrique » correspond le tenseur $(U+V)$ en entier. Ainsi l'hypothèse de la cohérence des pivotements des ions $\mathrm{NO}_{3}^{-}$n'apporte aucun changement dans les prévisions théoriques : elle n'a pas été envisagée par les auteurs hindous.

De même les pivotements des ions $\mathrm{CO}_{3}^{--}$de la calcite donnent naissance à des raies Raman de faible fréquence; la polarisation de l'une d'elles, $283 \mathrm{~cm}^{-1}$, a été soigneusement étudiée par J. Cabannes [8] et par Mye D. Osborne [9]. Nous avons reporté sur la figure 4 les résultats théoriques déduits du tenseur $(U+V)$ dans les six cas expérimentaux possibles suivant les orientations relatives du cristal, de la vibration incidente et du rayon diffusé. Les valeurs calculées du facteur de dépolarisation sont en excellent accord avec les résultats des mesures de $M^{110} D$. Osborne.

L'hypothèse des pivotements incohérents ne conduit qu'à une fréquence de pivotement. Or, dans la calcite, comme dans le nitrate de sodium, on observe deux raies Raman de faible fréquence et de forte intensité : 156 et $283 \mathrm{~cm}^{-1}$ pour la calcite, 98 et $185 \mathrm{~cm}^{-1}$ pour le nitrate de sodium. D'après Mlle D. Osborne, la raie $156 \mathrm{~cm}^{-1}$ présente sensiblement les mêmes caractères de polarisation que la raie $283 \mathrm{~cm}^{-1}$. On est donc amené à attribuer cette fréquence à une vibration de la maille dont le tenseur a la même forme que $(U+V)$, par exemple à des pivotements cohérents des deux ions autour d'axes de leur plan équatorial non plus parallèles, mais rectangulaires. On peut aussi songer à des pivotements cohérents autour d'axes inclinés à $120^{\circ}$, à cause de la symétrie ternaire du cristal. Cette hypothèse conduit à des tenseurs de même forme, mais a priori on doit prévoir alors deux fréquences supplémentaires suivant le mode de couplage des pivotements; il ne paraît pas qu'un tel dédoublement ait été observé jusqu'ici sur l'une des deux fréquences " externes " des ions $\mathrm{CO}_{3}^{--}$ou $\mathrm{NO}_{3}^{-}$.

5. Le pivotement des molécules dans les cristaux et l'origine de l'effet Cabannes-Daure dans les liquides. - On sait que dans le spectre de la diffusion moléculaire de la lumière par un liquide, la raie fondamentale est entourée d'un fond continu dépolarisé dont l'origine a été attribuée à la rotation des molécules (effet Cabannes-Daure). On explique ainsi son état de polarisation, la répartition des intensités avec la différence de fréquences, la variation de l'intensité globale avec l'anisotropie moléculaire et avec l'indice du liquide [r].

Cependant, Gross et Vuks [7], après leur découverte des raies Raman de faible fréquence de certains cristaux organiques (naphtalène, para-dibromobenzène, etc.) ont remarqué que ces composés donnent à l'état liquide un effet Cabannes-Daure important : ils en ont conclu que ces raies de faible fréquence caractérisent des liaisons intermoléculaires dans le réseau cristallin et que le spectre continu des liquides était encore dû à ces liaisons intermoléculaires déformées très fortement, mais se conservant tout de même à l'intérieur de la structure quasi cristalline des liquides. Le spectre continu des liquides ne serait pas dû à la rotation des molécules, mais d̀ un effet de vibration.

Pour Sirkar et ses collaborateurs [ro] au contraire, des fréquences d'oscillation linéaires du réseau cristallin ne peuvent avoir qu'exceptionnellement une intensité appréciable; il faut donc attribuer 
ces radiations à des oscillations intermoléculaires dans des groupes fortement polymérisés. Ces groupes disparaissent à la fusion et l'effet Cabannes-Daure est dû en majeure partie à la rotation des molécules dans le liquide. Cette hypothèse de Sirkar a pour origine la forte intensité des raies de faible fréquence, comparée à l'intensité des raies Raman internes. D'après notre interprétation, c'est aux forces d'orientation des molécules à l'intérieur du cristal, forces qui admettent les éléments de symétrie de la maille, qu'est due la cohérence des pivotements. Il faut remplacer l'hypothèse des groupements fortement polymérisés par l'idée des domaines élémentaires de cohérence dont l'étendue reste encore à préciser.

Pour Bhagavantam et ses collaborateurs [i I], comme pour Venkateswaran [1 2] les oscillations des molécules du cristal persistent dans le liquide à structure quasi cristalline, mais cessent d'être quantifiées; elles donnent un fond continu. Cependant, pour lier l'intensité de ce fond à l'anisotropie moléculaire, il faut supposer, qu'au cours de ces mouvements, l'orientation des molécules varie : ils envisagent donc des oscillations des molécules autour d'orientations moyennes imposées par le cristal ou, à son défaut, imposées par la structure quasi cristalline dans le liquide.

Nos mesures montrent nettement que dans un cristal organique, mou, à point de fusion bas comme le naphtalène, les pivotements cohérents des molécules se font autour de leurs axes principaux d'inertie pour donner naissance aux raies de faible fréquence découvertes par Gross et Vuks. Lorsque la température s'élève, l'amplitude de ces pivotements augmente et à la fusion, avec la disparition du réseau, ces pivotcments se transforment en rotations.

En effet, à la fusion, les forces d'orientation du réseau cristallin disparaissent et avec elles les forces de couplage des pivotements. Or, la forte intensité de la diffusion de fréquence modifiée, due aux fluctuations d'orientation des molécules. et qu'on retrouve dans l'effet Cabannes-Daure des liquides, ne peut s'expliquer que par la rotation complète des molécules : des pivotements incohérents n'ajouteraient à la diffusion Rayleigh non changée de longueur d'onde, qu'un faible fond continu [r]

L'intensité de l'effet de pivotement dans les cristaux comme celle de l'effet de rotation dans les liquides sont liées aux fluctuations d'orientation, donc à l'anisotropie optique des molécules diffusantes. Ces deux effets doivent donc être tous les deux particulièrement intenses avec les composés aromatiques dont l'anisotropie optique très élevée se révèle également par une biréfringence remarquable de leurs cristaux. L'existence de radiations de faible fréquence dans le spectre Raman des cristaux organiques n'apporte donc aucun argument en faveur de la persistance à l'état liquide d'une structure quasi cristalline, contrairement aux conclusions de Gross et Vuks, et, à leur suite, de certains auteurs hindous.

Nous sommes reconnaissants à M. J. Cabannes, Professeur à la Sorbonne, de l'intérêt qu'il nous a témoigné pendant ces recherches. Nous remercions notre collègue, M. Brus, Directeur de l'Institut du Pin, qui a mis à notre disposition une partie du matériel spectrographique de son laboratoire.

BIBLIOGRAPHIE.

[1] A. Rousset, Thèses, Paris, 1935; Annales de Physique, I 936,5, p. 6 o.

[2] C. V. Raman et T. M. K. Negundaki, Nature, 1939, 143, p. 679 .

[3] J. Cabannes, Comptes rendus, i94o, 211, p. 625.

[4] J. M. Robertson, Proc. Roy. Soc. London, A, r933, 142 , p. 674 .

[5] H. Benel, Thèses, Bordeaux, r940, p. 7 et planche I.

[6] A. Kastler et A. Rousset, Comples rendus, r 94 I, 212, p. 645 .

[7] E. Gross et M. Vuks, Nature, r935, 135, p. г оo, 43 r et $998 ;$ Journ. de Phys., I 935,6, p. 457 et r 936,7 , p. I 13.

[8] J. Cabannes, Comptes rendus, i929, 188, p. г 04 i ; Transactions of the Faraday Society, septembre $\times 9^{2} 9$, p. $82_{2} 3$.
[9] D. Osborne, Thèse d'Université, Montpellier, r 93 I.

[10] S. C. Sirkar, Indian Journal of Physics, 1936, 10, p. 75; S. C. Sirkar et B. S. Mookerjee, ibid., p. 375 ; S. C. Sirkar et J. Gupta, ibid., p. 473 , et i 937,11 , p. 55 et 283 ; S. C. Sirkar ibid., p. 343 ; S. C. Sirkar et I. C. Bishui, ibid., 1938, 11, p. 417; S. C. Sirkar et J. Gupta, ibid., 1938, 12, p. 35 .

[11] S. Bhagavantam, Proc. of the Indian Academy of Sciences, A, 1935, 2, p. 63; S. Bhagavantam et T. Venkatarayudu, ibid., 1939, 9, p. 224.

[12] C. S. Venkateswaran, Proc. of the Indian Academy of Sciences, A, r936, 4, p. 414; Current Science, 1938, 8, p. 378 . 